\title{
A CLASSIFICATION OF TRIGONAL RIEMANN SURFACES
}

\author{
Robert D. M. ACCOLA
}

\begin{abstract}
Necessary and sufficient conditions are given for the existence of a trigonal Riemann surface with given genus and types of ramification points.
\end{abstract}

A compact Riemann surface $W_{p}$ of genus $p(p>3)$ is said to be trigonal if it admits a three sheeted covering of the Riemann sphere. The fibers of this map form a linear series $g_{3}^{1}$. All non-hyperelliptic Riemann surfaces of genus 4 are trigonal and generically admit two distinct $g_{3}^{1}$ 's. Exceptionally, a nonhyperelliptic $W_{4}$ admits a unique $g_{3}^{1}$ which is half-canonical. For $p>4$ the locus of trigonal Riemann surfaces in moduli space has codimension $p-4$. Also for $p>4$ a trigonal Riemann surface has a unique $g_{3}^{1}$.

For a trigonal Riemann surface $W_{p}$, there is a unique integer $l$ so that the complete linear series $\left|(l) g_{3}^{1}\right|$ and $\left|(l+1) g_{3}^{1}\right|$ have dimensions $l$ and $l+\varepsilon$ respectively where $\varepsilon>1$. We shall call $l$ the trigonal index of $W_{p}$ and denote it tl. (Then $t i \leq p / 2$.)

Now we consider the ramification points of the three sheeted cover using the terminology introduced by Coppens. Ramification points of multiplicity two will be called ordinary, and those of multiplicity three will be called total. Ramification points occurring in the residual divisor (defined below), when $t i<$ $p / 2$, will be said to be of Type II, and otherwise of Type I. If $t l=p / 2$ then all ramification points are of Type $I$. Thus there are four kinds of ramification points.

Recently trigonal Riemann surfaces have been studied extensively in the context of the trigonal-tetragonal relation, but from the viewpoint of this paper the literature in more sparse. Despite the work of Hensel-Landsberg [4] concerning the dimension in moduli space of the loci of trigonal surfaces with given trigonal index, very little seems to have occurred until Coppens distinguished the four kinds of ramification points [2], [3]. Then Kato and Horiuchi [6], [5] derived a canonical algebraic equation of degree three defining a trigonal Riemann surface. This derivation for trigonal surfaces occurred more than a century after the corresponding derivation for hyperelliptic surfaces. It appears that the work

1991 Mathematics Subject Classification: $14 \mathrm{H}$.

Received March 8, 1999; revised June 30, 1999. 
of Coppens was necessary for this derivation to be done. In this paper we build upon the work of Coppens, Kato, and Horiuchi.

Now we quote without proof some results concerning the trigonal index. The proofs follow from the Riemann-Roch theorem. Also see [2], [3]. $\quad\left(K_{p}\right.$ is the canonical divisor.)

Lemma 1. Suppose that $W_{p}$ is a trigonal Riemann surface. Then

1) $(p-1-t i) g_{3}^{1}$ is the largest multiple of $g_{3}^{1}$ that is special.

2) $K_{p}=(p-1-t i) g_{3}^{1}+R$, where $|R|=g_{\rho}^{\varepsilon}, \varepsilon=0$ or 1 , and $\rho=3 t i-$ $(p-1) . \quad(R$ will be called the residual divisor $)$

i) $\varepsilon=0$ if and only if $t i<p / 2$.

ii) $\varepsilon=1$ if and only if $t i=p / 2$. In this case: $\rho=(p / 2)+1$.

3) $(p-1) / 3 \leq t i \leq p / 2$

LEMma 2. Suppose that $W_{p}$ is a trigonal Riemann surface. If $t i<$ $(p-1) / 2$, then ti is the unique integer so that $\left|(t i) g_{3}^{1}\right|$ and $\left|K_{p}-(t i) g_{3}^{1}\right|$ are both compounded of $g_{3}^{1}$.

If the trigonal index and genus are given then the Weierstrass weight of each of the four kinds of ramification points is easily computed. (See [6] or [2], [3].) The only ramification points which are not Weierstrass points occur when the trigonal index is maximum and the ramification points are ordinary and of Type I.

Now we outline some of the work of Kato-Horiuchi [6] and Kato [5]. Let $W_{p}$ be a trigonal Riemann surface of genus $p$ and trigonal index $l$. Then $W_{p}$ admits a meromorphic function $y$ of degree $3 l+3$ satisfying

$$
y^{3}+Q(x) y+R(x)=0
$$

where $Q$ and $R$ are polynomials, degree $Q=2 l+2$, degree $R=3 l+3$, and degree $\left(4 Q^{3}+27 R^{2}\right)=6 l+6$. The poles of $y$ all occur in the divisor of $g_{3}^{1}$ above $\infty$. To describe $Q$ and $R$ more precisely we have seven monic polynomials:

$\Pi_{1}, \Pi_{2}, \Pi_{3}, \Pi_{4}$ all with simple roots, with degrees $s, t, n, m$ and $\Gamma_{1}, \Gamma_{2}, \Gamma_{3}$ (not necessarily with simple roots) with degrees $\sigma, \mu, v$.

We also have a non-zero constant $\lambda$. Then $Q$ and $R$ are as follows:

$$
Q=\lambda \Gamma_{1} \Pi_{1}\left(\Pi_{2}\right)^{2} \Pi_{4} \quad R=(2 \lambda) /(3 \sqrt{3}) \Gamma_{2} \Pi_{1}\left(\Pi_{2}\right)^{2}\left(\Pi_{4}\right)^{2}
$$

Then $4 Q^{3}+27 R^{2}=4 \lambda^{2}\left(\Pi_{1}\right)^{2}\left(\Pi_{2}\right)^{4}\left(\Pi_{4}\right)^{3}\left[\lambda\left(\Gamma_{1}\right)^{3} \Pi_{1}\left(\Pi_{2}\right)^{2}+\left(\Gamma_{2}\right)^{2} \Pi_{4}\right]$. Now define three monic polynomials $P_{1}, P_{2}$, and $P_{3}$ as follows:

$$
\begin{gathered}
P_{2}=\left(\Gamma_{2}\right)^{2} \Pi_{4} \\
P_{3}=\left(\Gamma_{1}\right)^{3} \Pi_{1}\left(\Pi_{2}\right)^{2}
\end{gathered}
$$


Then $P_{1}$ is the monic polynomial so that

$$
(1+\lambda) P_{1}=P_{2}+\lambda P_{3}
$$

and

$$
P_{1}=\left(\Gamma_{3}\right)^{2} \Pi_{3}
$$

By considering the degrees of the various polynomials we have the following:

$$
\begin{aligned}
& 2 l+2=m+s+2 t+\sigma \\
& 3 l+3=2 m+s+2 t+\mu \\
& 6 l+6=3 m+2 s+4 t+n+2 v .
\end{aligned}
$$

By algebra it follows that: $l+1=m+\mu-\sigma$ and

$$
\begin{gathered}
n+2 v=m+2 \mu=s+2 t+3 \sigma \\
\operatorname{Deg} P_{1}=\operatorname{Deg} P_{2}=\operatorname{Deg} P_{3} .
\end{gathered}
$$

$P_{1}, P_{2}$, and $P_{3}$ are coprime in pairs as are $\Pi_{1}$ and $\Pi_{2}$.

$n$ is the number of ordinary Type I ramification points.

$m$ is the number of ordinary Type II ramification points.

$s$ is the number of total Type I ramification points.

$t$ is the number of total Type II ramification points.

These ideas of Kato and Horiuchi are the starting point for the following discussion.

Suppose that we have the equation

$$
(1+\lambda) P_{1}=P_{2}+\lambda P_{3}
$$

where $P_{1}, P_{2}$, and $P_{3}$ are monic polynomials, coprime in pairs, and each of the same degree $d$. $\lambda$ is a non-zero constant. Then we can find seven monic polynomials $\Pi_{1}, \Pi_{2}, \Pi_{3}, \Pi_{4}$ all with simple roots, with degrees $s, t, n, m$, and $\Gamma_{1}, \Gamma_{2}, \Gamma_{3}$ (not necessarily with simple roots) with degrees $\sigma, \mu, v$, so that

$$
\begin{gathered}
P_{1}=\left(\Gamma_{3}\right)^{2} \Pi_{3} \\
P_{2}=\left(\Gamma_{2}\right)^{2} \Pi_{4} \\
P_{3}=\left(\Gamma_{1}\right)^{3} \Pi_{1}\left(\Pi_{2}\right)^{2} .
\end{gathered}
$$

Then

$$
d=n+2 v=m+2 \mu=s+2 t+3 \sigma
$$

Define $l$ by the equation

$$
l+1=m+\mu-\sigma
$$


Then

$$
\begin{gathered}
2 l+2=m+s+2 t+\sigma \\
3 l+3=2 m+s+2 t+\mu \\
6 l+6=3 m+2 s+4 t+n+2 v .
\end{gathered}
$$

We now obtain a trigonal Riemann surface defined by the equation

$$
y^{3}+Q(x) y+R(x)=0 \quad(\operatorname{Deg} y=3 l+3)
$$

by first going to the Galois closure of the three sheeted covering of $\boldsymbol{P}^{1}$ as follows: ( $Q$ and $R$ are defined by equations (2))

i) Define a rational function $Y$ by

$$
Y=(1+\lambda) P_{1} / P_{2}
$$

$Y$ is of degree $d$ with branching over $0, \infty$, and 1 described by equations (4)

ii) $\sqrt{Y}$ defines a hyperelliptic Riemann surface $W_{q} \cdot(1-\sqrt{Y}) /(1+\sqrt{Y})$ is a meromorphic function, $Z$, on $W_{q}$.

iii) $Z^{1 / 3}(=U)$ defines a cyclic 3 -sheeted cover $W_{r} \rightarrow W_{q}$.

$$
U^{3}+U^{-3}=(2+2 Y) /(1-Y)
$$

iv) Let $D_{3}$ be the dihedral group of order 6. Then $W_{r}$ is a $D_{3}$ cover of $\boldsymbol{P}^{1}$.

$$
D_{3}=\left\langle U \rightarrow e^{(2 \pi i / 3)} U, U \rightarrow U^{-1}\right\rangle
$$

v) Let $T=U+U^{-1}$. Then:

$$
T^{3}-3 T=U^{3}+U^{-3}=(2+2 Y) /(1-Y) .
$$

$T$ is invariant under the involution $\phi$ on $W_{r}$, where $\phi U=U^{-1}$. Thus $T$ is a function on the trigonal Riemann surface $W_{p}=W_{r} /\langle\phi\rangle$ where $2 p-2=n+m+$ $2 s+2 t-6$

vi) Let $y=3 R /(T-1) Q$ (Kato's transformation [5]). The equation

now follows by computation.

$$
y^{3}+Q y+R=0
$$

vii) If $l \leq(p / 2)$ then $l$ is the trigonal index. (See [5], notice that this is not required in the above discussion.)

Following Kato [5] we make the following definition.

Definition. Given six non-negative integers, $l, n, m, s, t, p$, where $2 p-2=$ $n+m+2 s+2 t-6$, and $l \leq(p / 2)$, let $\operatorname{Trig}_{p}(l, n, m, s, t)$ be the locus in moduli space of trigonal Riemann surfaces of genus $p$ with trigonal index $l$, and

$n$ : ordinary ramification points of Type $\mathrm{I}$.

$m$ : ordinary ramification points of Type II.

$s:$ total ramification points of Type $\mathrm{I}$.

$t$ : total ramification points of Type II. 
THEOREM. Necessary and sufficient conditions on the non-negative integers $l, n, m, s, t, p$, in order that $\operatorname{Trig}_{p}(l, n, m, s, t)$ be non-empty are:

1) $2 p-2=n+m+2 s+2 t-6$.

2) There exist non-negative integers $v, \mu, \sigma$, so that equations (5) are satisfied.

3) $l \leq(p / 2)$.

Remark. Note that equations (4) follow and that $l+1=m+\mu-\sigma$.

Proof. The necessity is due to Kato and Horiuchi [6], and Kato [5]. Sufficiency of the conditions: The existence of polynomials $P_{1}, P_{2}$, and $P_{3}$ whose zeros are described by equations (4) is equivalent to the existence of a rational function $Y: P^{1} \rightarrow P^{1}$ with ramification over $0, \infty$, and 1 determined by equations (4). To find such a $Y$ we must find a homomorphism from the fundamental group of

$$
P^{1}-\{0, \infty, 1\}-\{p+\sigma \text { other points }\}
$$

into $S_{d}$, the symmetric group on $\{1,2, \ldots, d\}$ where the permutations for $0, \infty$, and 1 are chosen to have cycle structure according to equations (4), the permutations for the other points are 2-cycles (transpositions), and the image of the homomorphism is a transitive subgroup of $S_{d}$. This will insure that the corresponding covering of $P^{1}$ is connected and has genus 0 (by the Riemann Hurwitz formula.) The covering map of degree $d$ with this monodromy group will be the desired rational function, $Y$. ([8], [1])

To do this we first observe in $S_{d}$ the following.

If $d$ is even let $\alpha=(1,2)(3,4) \cdots(d-1, d)$, and let $\beta=(2,3)(4,5) \cdots$ $(d-2, d-1)$.

If $d$ is odd let $\alpha=(1,2)(3,4) \cdots(d-2, d-1)$, and let $\beta=(2,3)(4,5) \cdots$ $(d-1, d)$. $d-1$.

In both cases $\alpha \beta$ is a $d$-cycle and the number of 2-cycles in both $\alpha$ and $\beta$ is

We shall consider in detail the case where $d$ is even. The case where $d$ is odd is handled in an analogous manner.

Let $X=P^{1}-\left\{0, \infty, 1, a_{1}, a_{2}, \ldots, a_{p+\sigma}\right\}$. Let $\gamma_{0}, \gamma_{\infty}, \gamma_{1}, \alpha_{1}, \alpha_{2}, \ldots, \alpha_{p+\sigma}$ be paths "circling" the punctures in $P^{1}$. Their homotopy classes generate $\pi_{1}(X, \cdot)$ with one relation. We choose to write this relation

$$
\gamma_{0} \alpha_{1} \alpha_{2} \cdots \alpha_{n / 2} \gamma_{\infty} \alpha_{(n / 2)+1} \cdots \alpha_{((n+m) / 2)-1} \gamma_{1} \alpha_{(n+m) / 2} \cdots \alpha_{p+\sigma}=e
$$

(Remember $p+\sigma=2 d-2-v-\mu-t-2 \sigma$

$$
=(n / 2)+((m / 2)-1)+(s+t+\sigma-1) .
$$

For the homomorphism from $\pi_{1}(X, \cdot) \rightarrow S_{d}$, proceed as follows: 


$$
\begin{aligned}
& \gamma_{0} \rightarrow(1,2)(3,4) \cdots(2 v-1,2 v) \\
& \alpha_{1} \rightarrow(2 v+1,2 v+2) \\
& \alpha_{2} \rightarrow(2 v+3,2 v+4) \\
& \ldots \\
& \alpha_{n / 2} \rightarrow(2 v+n-1,2 v+n) \quad(2 v+n=d) \\
& \gamma_{\infty} \rightarrow(2,3)(4,5) \cdots(2 \mu, 2 \mu+1) \\
& \alpha_{(n / 2)+1} \rightarrow(2 \mu+2,2 \mu+3) \\
& \alpha_{(n / 2)+2} \rightarrow(2 \mu+4,2 \mu+5) \\
& \ldots \\
& \alpha_{((n+m) / 2)-1} \rightarrow(2 \mu+m-2,2 \mu+m-1) .
\end{aligned}
$$

Then the product of these permutations is a cycle of length $d$. For convenience assume this $d$-cycle is $(d, d-1, d-2, \ldots, 3,2,1):=\delta$. Finally, let $\gamma_{1} \rightarrow(1,2,3)$. $(4,5,6) \cdots(3 \sigma-2,3 \sigma-1,3 \sigma)(3 \sigma+1,3 \sigma+2)(3 \sigma+3,3 \sigma+4) \cdots(3 \sigma+2 t-1,3 \sigma+2 t)$. Then $\delta \gamma_{1} \rightarrow$ a cycle of length $d-t-2 \sigma(=s+t+\sigma) . \delta \gamma_{1}$ can be written as a product of $s+t+\sigma-12$-cycles. The images of the remaining $\alpha$ 's are chosen to give the inverse of $\delta \gamma_{1}$. This completes the proof when $d$ is even.

Remarks. 1) One sees that $p+\sigma$ gives the dimension for $\operatorname{Trig}_{p}(l, n, m, s, t)$ if $l<p / 2$.

2) One can have fewer "other points" with more complicated ramification above them. Also one could have more complicated ramification points above $0, \infty$, and 1. For example, over 1 a ramification point of multiplicity four would correspond to a factor common to $\Gamma_{1}$ and $\Pi_{1}$. But the resulting surfaces would lie on subvarieties of $\operatorname{Trig}_{p}(l, n, m, s, t)$.

3) If $l=p / 2$ there will be, presumably, a one dimensional family of such models for each trigonal surface, corresponding, in some manner, to the fact that the residual divisor in this case moves in a pencil.

4) By letting two of the transpositions above the "other points" run together and cancel, the cover $Y: \boldsymbol{P}^{1} \rightarrow \boldsymbol{P}^{1}$ becomes disconnected, so that one can see rather easily the reducible stable boundary points in the closure of $\operatorname{Trig}_{p}(l, n, m, s, t)$. If one of the transpositions runs into two points above, say, 0 then we obtain a connected stable boundary point.

5) If two components belong to a boundary point of $\operatorname{Trig}_{p}(l, n, m, s, t)$ then the condition $l \leq p / 2$ need no longer hold for both components. Thus one cannot avoid the problem of considering surfaces that result from the construction of $Y$ in the proof of the theorem where hypothesis 3) need not hold. By naive dimension considerations one sees that generically such surfaces will have maximum trigonal index and all ramification points will be of Type $I$. $(s+t$ will still count the number of total ramification points [4].) 
6) The problem still remains of analyzing trigonal surfaces arising from the construction in the theorem when $l>p / 2$. This appears to be a difficult problem requiring a much closer look at the polynomials $P_{1}, P_{2}$, and $P_{3}$.

Note on terminology. The author has used the term trigonal index for what is sometimes referred to as the Maroni index (if it is one more than the index that Maroni in fact used). The reason for this is that an analysis deeper than that of Maroni (1946) [7] occurs in the book by Hensel-Landsberg (1902) [4, Chapter 31]. They compute the dimension in moduli space of trigonal surfaces with arbitrary trigonal index, and in doing so put their finger on a key parameter, which is here called $v$. At this point it appears futile to name the index after all three authors, so the neutral term is preferred.

\section{REFERENCES}

[ 1 ] Accola, R. D. M., Topics in the theory of Riemann surfaces, Lecture Notes in Math., 1595, Springer Verlag, 1991.

[2] Coppens, M., The Weierstrass gap sequence of the total ramification points of trigonal coverings of $P^{1}$, Indag. Math., 47 (1985), pp. 245-270.

[3] Coppens, M., The Weierstrass gap sequence of the ordinary ramification points of trigonal coverings of $P^{1}$; existence of a kınd of Weierstrass gap sequence, J. Pure Appl. Algebra, 43 (1986), pp. 11-25.

[4] Hensel, K. AND Landsberg, G., Theorie der algebraischen Funktionen einer Variablen und ihre Anwendung auf algebraische Kurven und Abelsche Integrale, Chelsea, New York, 1965, (Leipzig, 1902).

[ 5] Kato, T., Subspaces of trigonal Riemann surfaces, Koda1 Math. J., 12 (1989), pp. $72-91$.

[6] Kato, T. AND HoRiuchi, R., Weierstrass gap sequences at the ramification points of trigonal Riemann surfaces, J. Pure Appl. Algebra, 50 (1988), pp. 271-285.

[7] Maroni, A., Le serıe linearı speciali sulle curve trigonali, Ann. Mat. Pura Appl. (4), 25 (1946), pp. 343-354.

[ 8 ] Seifert, H. and Threlfall, W., Lehrbuch der Topologie, Chelsea, New York, 1945, (B. G. Teubner, Leipzig, 1934).

Department of Mathematics

BROWN UNIVERSITY

Providence, R. I. 02912

E-mail: rdma@brownvm.brown.edu 